\title{
Modified logarithmic Sobolev inequalities in null curvature
}

Ivan Gentil, Arnaud Guillin and Laurent Miclo

\begin{abstract}
We present a new logarithmic Sobolev inequality adapted to a log-concave measure on $\mathbb{R}$ between the exponential and the Gaussian measure. More precisely, assume that $\Phi$ is a symmetric convex function on $\mathbb{R}$ satisfying $(1+\varepsilon) \Phi(x) \leqslant x \Phi^{\prime}(x) \leqslant(2-\varepsilon) \Phi(x)$ for $x \geqslant 0$ large enough and with $\varepsilon \in] 0,1 / 2]$. We prove that the probability measure on $\mathbb{R} \mu_{\Phi}(d x)=e^{-\Phi(x)} / Z_{\Phi} d x$ satisfies a modified and adapted logarithmic Sobolev inequality: there exist three constants $A, B, C>0$ such that for all smooth functions $f>0$,
\end{abstract}

$$
\text { Ent }_{\mu_{\Phi}}\left(f^{2}\right) \leqslant A \int H_{\Phi}\left(\frac{f^{\prime}}{f}\right) f^{2} d \mu_{\Phi}
$$

with

$$
H_{\Phi}(x)=\left\{\begin{aligned}
x^{2} & \text { if }|x|<C \\
\Phi^{*}(B x) & \text { if }|x| \geqslant C
\end{aligned}\right.
$$

where $\Phi^{*}$ is the Legendre-Fenchel transform of $\Phi$.

\section{Introduction}

A probability measure $\mu$ on $\mathbb{R}^{n}$ satisfies a logarithmic Sobolev inequality if there exists $C \geqslant 0$ such that, for every smooth enough functions $f$ on $\mathbb{R}^{n}$,

$$
\operatorname{Ent}_{\mu}\left(f^{2}\right) \leqslant C \int|\nabla f|^{2} d \mu
$$

where

$$
\operatorname{Ent}_{\mu}\left(f^{2}\right):=\int f^{2} \log f^{2} d \mu-\int f^{2} d \mu \log \int f^{2} d \mu
$$

and where $|\nabla f|$ is the Euclidean length of the gradient $\nabla f$ of $f$.

2000 Mathematics Subject Classification: 26D99, 60E15, 39B72.

Keywords: Logarithmic Sobolev inequality, Poincaré inequality, concentration inequality, log-concave measure. 
Gross in [9] defines this inequality and shows that the canonical Gaussian measure with density $(2 \pi)^{-n / 2} e^{-|x|^{2} / 2}$ with respect to the Lebesgue measure on $\mathbb{R}^{n}$ is the basic example of measure $\mu$ satisfying (1.1) with the optimal constant $C=2$. Since then, many results have presented measures satisfying such an inequality, among them the famous Bakry-Émery $\boldsymbol{\Gamma}_{\mathbf{2}}$-criterion, that we recall now in our particular case. Let $\Phi$ a $\mathcal{C}^{2}$ function on $\mathbb{R}^{n}$ and note $\mu_{\Phi}(d x)=\exp (-\Phi(x)) / Z_{\Phi} d x, Z_{\Phi}$ being the normalization constant so that $\mu_{\Phi}$ is a probability measure on $\mathbb{R}^{n}$. Assume that there exists $\lambda>0$ such that

$$
\forall x \in \mathbb{R}^{n}, \quad \operatorname{Hess}(\Phi(x)) \geqslant \lambda \mathrm{Id}
$$

in the sense of symmetric matrix. Then Bakry and Émery proved that $\mu$ is satisfying inequality (1.1) with an optimal constant $C \in[0,2 / \lambda]$. We refer to $[3,2]$ for the $\boldsymbol{\Gamma}_{\mathbf{2}}$-criterion and to $[1,10]$ for a review on logarithmic Sobolev inequality.

The interest of this paper is to give a logarithmic Sobolev inequality when the probability measure $\mu_{\Phi}$ on $\mathbb{R}$ defined before does not satisfy (1.2) but $\Phi^{\prime \prime}(x) \geqslant 0, \forall x \in \mathbb{R}$. A first answer is given for the following particular measure: let $\alpha \geqslant 1$ and define the probability measure $\mu_{\alpha}$ on $\mathbb{R}$ by

$$
\mu_{\alpha}(d x)=\frac{1}{Z_{\alpha}} e^{-|x|^{\alpha}} d x
$$

where $Z_{\alpha}=\int e^{-|x|^{\alpha}} d x$.

The authors prove, in [8], that for $1<\alpha<2$, the measure $\mu_{\alpha}$ satisfies the following inequalities, for all smooth functions such that $f \geqslant 0$ and $\int f^{2} d \mu_{\alpha}=1$

$$
\operatorname{Ent}_{\mu_{\alpha}}\left(f^{2}\right) \leqslant A \operatorname{Var}_{\mu_{\alpha}}(f)+B \int_{f \geqslant 2}\left|\frac{f^{\prime}}{f}\right|^{\beta} f^{2} d \mu_{\alpha},
$$

where $A$ and $B$ are some constants, $\alpha^{-1}+\beta^{-1}=1$ and

$$
\operatorname{Var}_{\mu_{\alpha}}(f):=\int f^{2} d \mu_{\alpha}-\left(\int f d \mu_{\alpha}\right)^{2}
$$

It is well-known that the probability measure $\mu_{\alpha}$ satisfies (still for $\alpha \geqslant 1$ ) a Poincaré inequality (or spectral gap inequality), i.e. for every smooth enough function $f$,

$$
\operatorname{Var}_{\mu_{\alpha}}(f) \leqslant C \int|\nabla f|^{2} d \mu_{\alpha}
$$

where $0<C<\infty$. 
Then using (1.5) and (1.4) we get that $\mu_{\alpha}$ satisfies also this modified logarithmic Sobolev inequality for all smooth and positive function $f$,

$$
\text { Ent }_{\mu}\left(f^{2}\right) \leqslant C \int H_{a, \alpha}\left(\frac{f^{\prime}}{f}\right) f^{2} d \mu
$$

here and in the whole paper the convention that $0 \cdot \infty=0$ is assumed, otherwise stated where $a$ and $C$ are positive constants and

$$
H_{a, \alpha}(x)= \begin{cases}x^{2} & \text { if }|x|<a, \\ |x|^{\beta} & \text { if }|x| \geqslant a,\end{cases}
$$

with $1 / \alpha+1 / \beta=1$. This version of logarithmic Sobolev inequality admits a $n$ dimensional version, for all smooth function $f$ on $\mathbb{R}^{n}$,

$$
\text { Ent }_{\mu_{\alpha}^{\otimes n}}\left(f^{2}\right) \leqslant C \int H_{a, \alpha}\left(\frac{\nabla f}{f}\right) f^{2} d \mu_{\alpha}^{\otimes n},
$$

where by definition we have taken

$$
H_{a, \alpha}\left(\frac{\nabla f}{f}\right):=\sum_{i=1}^{n} H_{a, \alpha}\left(\frac{\partial_{i} f}{f}\right) .
$$

Note that Bobkov and Ledoux gave in [7] a corresponding result for the critical (exponential) case, when $\alpha=1$.

Our main purpose here will be to establish the generalization of inequalities (1.4), (1.6) and (1.7) when the measure on $\mathbb{R}$ is only a log-concave measure between $e^{-|x|}$ and $e^{-x^{2}}$. More precisely, let $\Phi$ be a function on $\mathbb{R}$, we say that $\Phi$ satisfies the property $(\mathbf{H})$ if this two properties are satisfied:

- $\Phi$ is a $\mathcal{C}^{2}$, symmetric and strictly convex on $\mathbb{R}$.

- There exits $M>0$ and $0<\varepsilon \leqslant 1 / 2$ such that $\Phi(M)>0$ and

$$
\forall x \geqslant M, \quad(1+\varepsilon) \Phi(x) \leqslant x \Phi^{\prime}(x) \leqslant(2-\varepsilon) \Phi(x) .
$$

We assume along the article that the function $\Phi$ on $\mathbb{R}$ verifies hypothesis $(\mathbf{H})$.

Remark 1.1 The assumption $(\mathbf{H})$ implies that there exists $m_{1}, m_{2}>0$ such that

$$
\forall x \geqslant M, \quad m_{1} x^{1 /(1-\varepsilon)} \leqslant \Phi(x) \leqslant m_{2} x^{2-\varepsilon} .
$$

This remark explains why, under the hypothesis $(\mathbf{H})$, the function $\Phi$ lies between $e^{-|x|}$ and $e^{-x^{2}}$.

Due to Remark 1.1, $\int e^{-\Phi(x)} d x<\infty$. Then we define the probability measure $\mu_{\Phi}$ on $\mathbb{R}$ by

$$
\mu_{\Phi}(d x)=\frac{1}{Z_{\Phi}} e^{-\Phi(x)} d x
$$

where $Z_{\Phi}=\int e^{-\Phi(x)} d x$. 
The main result of this article is the following theorem:

Theorem 1.2 Assume $(\mathbf{H})$ then there exist constants $A, A^{\prime}, B, D \geqslant 0$ and $\kappa>0$ such that for any smooth functions $f \geqslant 0$ satisfying $\int f^{2} d \mu_{\Phi}=1$ we have

$$
\operatorname{Ent}_{\mu_{\Phi}}\left(f^{2}\right) \leqslant A \operatorname{Var}_{\mu_{\Phi}}(f)+A^{\prime} \int_{f^{2} \geqslant \kappa} H_{\Phi}\left(\frac{f^{\prime}}{f}\right) f^{2} d \mu_{\Phi}
$$

where

$$
H_{\Phi}(x)=\left\{\begin{aligned}
x^{2} & \text { if }|x|<D \\
\Phi^{*}(B x) & \text { if }|x| \geqslant D
\end{aligned}\right.
$$

where $\Phi^{*}$ is the Legendre-Fenchel transform of the function $\Phi, \Phi^{*}(x):=$ $\sup _{y \in \mathbb{R}}\{x \cdot y-\Phi(y)\}$.

It is well known that the measure $\mu_{\Phi}$ satisfies a Poincaré inequality (inequality (1.5) for the measure $\mu_{\Phi}$, see for example Chapter 6 of [1]). Then we obtain the following corollary:

Corollary 1.3 Let $\Phi$ satisfying the property $(\mathbf{H})$ then there exists $A, B, D \geqslant 0$ such that for any smooth functions $f>0$ we have

$$
\text { Ent }_{\mu_{\Phi}}\left(f^{2}\right) \leqslant A \int H_{\Phi}\left(\frac{f^{\prime}}{f}\right) f^{2} d \mu_{\Phi}
$$

where $H_{\Phi}$ is defined on (1.10).

In [8] we investigate some particular example, where we have $\Phi(x)=$ $|x|^{\alpha} \log ^{\beta}|x|$, for $\left.\alpha \in\right] 1,2[$ and $\beta \in \mathbb{R}$. Theorem 1.2 gives the result in the general case.

Definition 1.4 Let $\mu$ a probability measure on $\mathbb{R}^{n} \cdot \mu$ satisfies a Modified Logarithmic Sobolev Inequality (MLSI) of function $H_{\Phi}$ (defined on (1.10)) if there exists $A \geqslant 0$ such that for any smooth functions $f>0$ we have

$$
\text { Ent }_{\mu_{\Phi}}\left(f^{2}\right) \leqslant A \int H_{\Phi}\left(\frac{\nabla f}{f}\right) f^{2} d \mu_{\Phi},
$$

where

$$
H_{\Phi}\left(\frac{\nabla f}{f}\right):=\sum_{i=1}^{n} H_{\Phi}\left(\frac{\partial_{i} f}{f}\right) .
$$

The $M L S I$ of function $H_{\Phi}$ is the $n$-dimensional version of inequality (1.11). 
In Section 2 we will give the proof of Theorem 1.2. The proof is done in two steps, Proposition 2.4 and 2.9. In Subsection 2.1, we will describe the case where the entropy is large and in Subsection 2.2 we will study the other case, when the entropy is small. This two cases exhibit very different behavior as we will see in the next sections but they are connected via the common use of Hardy's inequality we recall now.

Let $\mu, \nu$ be Borel measures on $\mathbb{R}^{+}$. Then the best constant $A$ so that every smooth functions $f$ satisfy

$$
\int_{0}^{\infty}(f(x)-f(0))^{2} d \mu(x) \leqslant A \int_{0}^{\infty} f^{\prime 2} d \nu
$$

is finite if and only if

$$
B=\sup _{x>0}\left\{\mu \left(\left[x, \infty[) \int_{0}^{x}\left(\frac{d \nu^{a c}}{d t}\right)^{-1} d t\right\}\right.\right.
$$

is finite, where $\nu^{a c}$ is the absolutely continuous part of $\nu$ with respect to $\mu$. Moreover, we have (even if $A$ or $B$ is infinite),

$$
B \leqslant A \leqslant 4 B
$$

We refer to $[12]$ or $[6,1]$ for a review in this domain.

In Section 3 we will explain some classical properties of this particular logarithmic Sobolev inequality. We explain briefly how, as in the classical logarithmic Sobolev inequality of Gross,

- the $M L S I$ of function $H_{\Phi}$ satisfies the tensorization and the perturbation properties,

- the $M L S I$ of function $H_{\Phi}$ implies also Poincaré inequality.

The last application is the concentration property for probability measure satisfying inequality (1.11). Indeed, we obtain Hoeffding's type inequality: assume that a measure $\mu$ on $\mathbb{R}$ satisfies inequality (1.11) and let $f$ be a Lipschitz function on $\mathbb{R}$ with $\|f\|_{\text {Lip }} \leqslant 1$, then, for some constants $A, B, D \geqslant 0$ independent of the dimension $n$,

$$
\begin{aligned}
\mathbb{P}\left(\frac{1}{n} \mid \sum_{k=1}^{n} f\left(X_{k}\right)-\right. & \mu(f) \mid>\lambda) \\
& \leqslant \begin{cases}2 \exp (-n A \Phi(B \lambda)) & \text { if } \lambda \geqslant D, \\
2 \exp \left(-n A \lambda^{2}\right) & \text { if } 0 \leqslant \lambda \leqslant D,\end{cases}
\end{aligned}
$$


or equivalently,

$$
\begin{aligned}
\mathbb{P}\left(\frac{1}{\sqrt{n}}\left|\sum_{k=1}^{n} f\left(X_{k}\right)-\mu(f)\right|>\lambda\right) \leqslant & \begin{cases}2 \exp \left(-n A \Phi\left(B \frac{\lambda}{\sqrt{n}}\right)\right) & \text { if } \lambda \geqslant D \sqrt{n} \\
2 \exp \left(-A \lambda^{2}\right) & \text { if } 0 \leqslant \lambda \leqslant D \sqrt{n}\end{cases}
\end{aligned}
$$

Inequality (1.15) is interesting as for large enough $n$ we find the Gaussian concentration, this is natural due to the convergence of

$$
\frac{1}{\sqrt{n}}\left(\sum_{k=1}^{n} f\left(X_{k}\right)-\mu(f)\right)
$$

to the Gaussian distribution. This result is not a new one, Talagrand explains it in [13], see also [11] for a large review on this topic, the interest of this part is to observe this phenomenon via Logarithmic Sobolev inequality. Note also that it implies control of Laplace functionals which have applications for example in statistics.

Note finally that Barthe, Cattiaux and Roberto [4] have studied the same sort of log-concave measure, they prove functional inequalities with an other point of view, namely Beckner type inequalities or $\Phi$-Sobolev inequalities, in particular one of their results is concentration inequalities for the same measure $\mu_{\Phi}$.

\section{Proof of the Modified logarithmic Sobolev inequality (Theorem 1.2)}

Let us first give a lemma stating classical properties satisfied by the function $\Phi$ under $(\mathbf{H})$.

Lemma 2.1 Assume that $\Phi$ satisfies assumption $(\mathbf{H})$ then there exists $C \geqslant 0$ such that for large enough $x \geqslant 0$,

$$
\begin{gathered}
x^{2} \leqslant C \Phi^{*}(x), \\
\varepsilon \Phi\left(\Phi^{\prime-1}(x)\right) \leqslant \Phi^{*}(x) \leqslant(1-\varepsilon) \Phi\left(\Phi^{\prime-1}(x)\right), \\
\frac{1}{C} \Phi^{\prime-1}(x) \leqslant \frac{\Phi^{*}(x)}{x} \leqslant C \Phi^{\prime-1}(x) .
\end{gathered}
$$

The proof of Lemma 2.1 is an easy consequence of the property $(\mathbf{H})$.

For this we will note by smooth function a locally absolutely continuous function on $\mathbb{R}$. This is the regularity needed for the use of Hardy inequality in our case. 


\subsection{Large entropy}

The proof of $M L S I$ for large entropy is based on the next lemma, we give a MLSI saturate on the left, with some weighted type energy.

Lemma 2.2 There exists $C_{h} \geqslant 0$ and $M>0$ such that for every smooth functions $g$ we have

$$
\operatorname{Ent}_{\mu_{\Phi}}\left(g^{2}\right) \leqslant C_{h} \int g^{\prime 2} h d \mu_{\Phi}
$$

where $h$ is defined as follows

$$
h(x)=\left\{\begin{aligned}
1 & \text { if }|x|<M \\
\frac{x^{2}}{\Phi(x)} & \text { if }|x| \geqslant M .
\end{aligned}\right.
$$

Proof. We use Theorem 3 of [5] which is a refinement of the criterion of a Bobkov-Götze theorem (see Theorem 5.3 of [6]).

The constant $C_{h}$ satisfies

$$
\max \left(b_{-}, b_{+}\right) \leqslant C_{h} \leqslant \max \left(B_{-}, B_{+}\right)
$$

where

$$
\begin{aligned}
& b_{+}=\sup _{x \geqslant 0} \mu_{\Phi}\left(\left[x,+\infty[) \log \left(1+\frac{1}{2 \mu_{\Phi}([x,+\infty[)}\right) \int_{0}^{x} Z_{\Phi} \frac{e^{\Phi(t)}}{h(t)} d t\right.\right. \\
& \left.\left.b_{-}=\sup _{x \leqslant 0} \mu_{\Phi}(]-\infty, x\right]\right) \log \left(1+\frac{1}{\left.\left.2 \mu_{\Phi}(]-\infty, x\right]\right)}\right) \int_{x}^{0} Z_{\Phi} \frac{e^{\Phi(t)}}{h(t)} d t, \\
& B_{+}=\sup _{x \geqslant 0} \mu_{\Phi}\left(\left[x,+\infty[) \log \left(1+\frac{e^{2}}{\mu_{\Phi}([x,+\infty[)}\right) \int_{0}^{x} Z_{\Phi} \frac{e^{\Phi(t)}}{h(t)} d t,\right.\right. \\
& \left.\left.B_{-}=\sup _{x \leqslant 0} \mu_{\Phi}(]-\infty, x\right]\right) \log \left(1+\frac{e^{2}}{\mu_{\Phi}([-\infty, x[)}\right) \int_{x}^{0} Z_{\Phi} \frac{e^{\Phi(t)}}{h(t)} d t .
\end{aligned}
$$

An easy approximation proves that for large positive $x$

$$
\mu_{\Phi}\left(\left[x, \infty[)=\int_{x}^{\infty} \frac{1}{Z_{\Phi}} e^{-\Phi(t)} d t \sim_{\infty} \frac{1}{Z_{\Phi} \Phi^{\prime}(x)} e^{-\Phi(x)},\right.\right.
$$

and

$$
\int_{0}^{x} Z_{\Phi} \frac{e^{\Phi(t)}}{h(t)} d t \sim_{\infty} \frac{Z_{\Phi}}{h(x) \Phi^{\prime}(x)} e^{\Phi(x)}
$$

and one may prove similar behaviors for negative $x$. 
Then, there is $K$ and $M$ such that for $x \geqslant M$,

$$
\begin{aligned}
& \mu_{\Phi}\left(\left[x,+\infty[) \log \left(1+\frac{1}{2 \mu_{\Phi}([x,+\infty[)}\right) \int_{0}^{x} Z_{\Phi} \frac{e^{\Phi(t)}}{h(t)} d t \leqslant\right.\right. \\
& K \frac{\Phi(x)}{\Phi^{\prime}(x)^{2} h(x)}=K\left(\frac{\Phi(x)}{x \Phi^{\prime}(x)}\right)^{2} .
\end{aligned}
$$

The right hand term is bounded by the assumption $(\mathbf{H})$.

A simple calculation then yields that constants $b_{+}, b_{-}, B_{+}$and $B_{-}$are finite and the lemma is proved.

Remark 2.3 Note that this lemma can be proved in a more general case, when $\Phi$ does not satisfy hypothesis $(\mathbf{H})$.

Proposition 2.4 There exist $A, B, D, A^{\prime} \geqslant 0$ such that for any functions $f \geqslant 0$ satisfying

$$
\int f^{2} d \mu_{\Phi}=1 \text { and } \operatorname{Ent}_{\mu_{\Phi}}\left(f^{2}\right) \geqslant 1
$$

we have

$$
\operatorname{Ent}_{\mu_{\Phi}}\left(f^{2}\right) \leqslant A^{\prime} \operatorname{Var}_{\mu_{\Phi}}(f)+A \int_{f^{2} \geqslant 2} H_{\Phi}\left(\frac{f^{\prime}}{f}\right) f^{2} d \mu_{\Phi}
$$

where

$$
H_{\Phi}(x)=\left\{\begin{aligned}
x^{2} & \text { if }|x|<D \\
\Phi^{*}(B x) & \text { if }|x| \geqslant D .
\end{aligned}\right.
$$

As we will see in the proof, $A^{\prime}$ does not depend on the function $\Phi$.

Proof of Proposition 2.4. Let $f \geqslant 0$ satisfying $\int f^{2} d \mu_{\Phi}=1$. A careful study of the function

$$
x \rightarrow-x^{2} \log x^{2}+5(x-1)^{2}+x^{2}-1+(x-2)_{+}^{2} \log (x-2)_{+}^{2}
$$

proves that for every $x \geqslant 0$

$$
x^{2} \log x^{2} \leqslant 5(x-1)^{2}+x^{2}-1+(x-2)_{+}^{2} \log (x-2)_{+}^{2} .
$$

We know that $\int(f-1)^{2} d \mu_{\Phi} \leqslant 2 \operatorname{Var}_{\mu_{\Phi}}(f)$, recalling that $\int f^{2} d \mu_{\Phi}=1$ and $f \geqslant 0$,

$$
\begin{aligned}
\int f^{2} \log f^{2} d \mu_{\Phi} \leqslant 5 \int(f-1)^{2} d \mu_{\Phi}+\int\left(f^{2}-1\right) d \mu_{\Phi} \\
+\int(f-2)_{+}^{2} \log (f-2)_{+}^{2} d \mu_{\Phi} \\
\leqslant 10 \operatorname{Var}_{\mu_{\Phi}}(f)+\int(f-2)_{+}^{2} \log (f-2)_{+}^{2} d \mu_{\Phi} .
\end{aligned}
$$


Since $\int f^{2} d \mu_{\Phi}=1$, one can easily prove that

$$
\int(f-2)_{+}^{2} d \mu_{\Phi} \leqslant 1
$$

then $\int(f-2)_{+}^{2} \log (f-2)_{+}^{2} d \mu_{\Phi} \leqslant$ Ent $_{\mu_{\Phi}}\left((f-2)_{+}^{2}\right)$, and

$$
\text { Ent }_{\mu_{\Phi}}\left(f^{2}\right) \leqslant 10 \operatorname{Var}_{\mu_{\Phi}}(f)+\text { Ent }_{\mu_{\Phi}}\left((f-2)_{+}^{2}\right) \text {. }
$$

Lemma 2.2 with $g=(f-2)_{+}$gives

$$
\text { Ent }_{\mu_{\Phi}}\left((f-2)_{+}^{2}\right) \leqslant C_{h} \int(f-2)_{+}^{\prime 2} h d \mu_{\Phi}=C_{h} \int_{f \geqslant 2} f^{\prime 2} h d \mu_{\Phi} .
$$

Due to the assumption $(\mathbf{H})$, the function $h(x)=x^{2} / \Phi(x)$, is increasing on $[M, \infty[$ and

$$
\lim _{x \rightarrow \infty} h(x)=\infty
$$

We can assume that $\Phi(M)>0$. We note $m=h(M)>0$ Let us define the function $\tau$ as follow

$$
\tau(x)=\left\{\begin{array}{lr}
x \Phi\left(h^{-1}(m)\right) /\left(8 C_{h} m\right) & \text { if } 0 \leqslant x \leqslant m \\
\Phi\left(h^{-1}(x)\right) /\left(8 C_{h}\right) & \text { if } x \geqslant m
\end{array}\right.
$$

For all $x \geqslant M$, we have $\tau(h(x))=\Phi(x) /\left(8 C_{h}\right)$ and then, an easy calculus gives that $\tau$ is increasing on $[0, \infty[$.

Let $u>0$,

$$
\begin{aligned}
C_{h} \int_{f \geqslant 2} f^{\prime 2} h d \mu_{\Phi} & =C_{h} \int_{f \geqslant 2} u\left(\frac{f^{\prime}}{f}\right)^{2} \frac{h}{u} f^{2} d \mu_{\Phi} \\
& \leqslant C_{h} \int_{f \geqslant 2} \tau^{*}\left\{u\left(\frac{f^{\prime}}{f}\right)^{2}\right\} f^{2} d \mu_{\Phi}+\int_{f \geqslant 2} C_{h} \tau\left(\frac{h}{u}\right) f^{2} d \mu_{\Phi}
\end{aligned}
$$

For every function $f$ such that $\int f^{2} d \mu_{\Phi}=1$ and for every measurable function $g$ such that $\int f^{2} g d \mu_{\Phi}$ exists we get

$$
\int f^{2} g d \mu \leqslant \operatorname{Ent}_{\mu_{\Phi}}\left(f^{2}\right)+\log \int e^{g} d \mu_{\Phi} .
$$

Indeed, this inequality is also true for all function $g \geqslant 0$ even if the above integrals are infinite.

We apply the previous inequality with $g=4 C_{h} \tau(h / u)$ and we obtain

$$
\begin{aligned}
\int_{f \geqslant 2} C_{h} \tau\left(\frac{h}{u}\right) f^{2} d \mu_{\Phi} \leqslant \frac{1}{4} \int 4 C_{h} \tau & \left(\frac{h}{u}\right) f^{2} d \mu_{\Phi} \leqslant \\
& \frac{1}{4}\left(\operatorname{Ent}_{\mu_{\Phi}}\left(f^{2}\right)+\log \int e^{4 C_{h} \tau\left(\frac{h}{u}\right)} d \mu_{\Phi}\right) .
\end{aligned}
$$


If $u=1$ we have, by construction, $\int e^{4 C_{h} \tau\left(\frac{h}{u}\right)} d \mu_{\Phi}<\infty$, then we get

$$
\lim _{u \rightarrow \infty} \int e^{4 C_{h} \tau\left(\frac{h}{u}\right)} d \mu_{\Phi}=1
$$

Then, by the bounded convergence theorem, there exists $u_{0}$ such that we get $\int e^{4 C_{h} \tau\left(\frac{h}{u_{0}}\right)} d \mu_{\Phi} \leqslant e$.

Thus we have

$$
\text { Ent }_{\mu_{\Phi}}\left((f-2)_{+}{ }^{2}\right) \leqslant C_{h} \int_{f \geqslant 2} \tau^{*}\left\{u_{0}\left(\frac{f^{\prime}}{f}\right)^{2}\right\} f^{2} d \mu_{\Phi}+\frac{1}{4} \mathbf{E n t}_{\mu_{\Phi}}\left(f^{2}\right)+\frac{1}{4}
$$

Ent $_{\mu_{\Phi}}\left(f^{2}\right) \geqslant 1$, implies

$$
\text { Ent }_{\mu_{\Phi}}\left(f^{2}\right) \leqslant 20 \operatorname{Var}_{\mu_{\Phi}}(f)+2 C_{h} \int_{f \geqslant 2} \tau^{*}\left\{u_{0}\left(\frac{f^{\prime}}{f}\right)^{2}\right\} f^{2} d \mu_{\Phi} .
$$

Then Lemma 2.5 gives the proof of inequality (2.7).

Lemma 2.5 There exist constants $A, B, C, D \geqslant 0$ such that

$$
\forall x \geqslant 0, \quad \tau^{*}\left(x^{2}\right) \leqslant \begin{cases}B x^{2} & \text { if } x<D \\ A \Phi^{*}(C x) & \text { if } x \leqslant D .\end{cases}
$$

Proof. Let $x>0$, then $\tau^{*}(x)=\sup _{y \geqslant 0}\{x y-\tau(y)\}$. Let now $m=h(M)>0$, then

$$
\begin{aligned}
\tau^{*}(x) & =\max \left\{\sup _{y \in[0, m[}\{x y-\tau(y)\}, \sup _{y \geqslant m}\{x y-\tau(y)\}\right\}, \\
& \leqslant \sup _{y \in[0, m[}\{x y-\tau(y)\}+\sup _{y \geqslant m}\{x y-\tau(y)\} .
\end{aligned}
$$

We have $\sup _{y \in[0, m[}\{x y-\tau(y)\} \leqslant x m$, because $\tau$ is positive. Then the definition of $\tau$ implies that

$$
\sup _{y \geqslant m}\{x y-\tau(y)\}=\sup _{y \geqslant M}\left\{x \frac{y^{2}}{\Phi(y)}-\frac{\Phi(y)}{8 C_{h}}\right\} .
$$

Let define $\psi_{x}(y)=x y^{2} / \Phi(y)-\Phi(y) /\left(8 C_{h}\right)$ for $y \geqslant M$. We have

$$
\psi_{x}^{\prime}(y)=x y \frac{2 \Phi(y)-y \Phi^{\prime}(y)}{\Phi^{2}(y)}-\frac{\Phi^{\prime}(y)}{8 C_{h}} .
$$

Due to the property $(\mathbf{H})$, there is $D>0$ such that

$$
\forall x \geqslant D, \quad \sup _{y \geqslant m}\{x y-\tau(y)\}=x \frac{y_{x}^{2}}{\Phi\left(y_{x}\right)}-\frac{\Phi\left(y_{x}\right)}{8 C_{h}},
$$


where $y_{x} \geqslant M$ satisfies

$$
x=\frac{1}{8 C_{h}} \frac{\Phi^{\prime}\left(y_{x}\right) \Phi^{2}\left(y_{x}\right)}{y_{x}\left(2 \Phi\left(y_{x}\right)-y_{x} \Phi^{\prime}\left(y_{x}\right)\right)} .
$$

The assumption $(\mathbf{H})$ implies that

$$
\varepsilon y_{x} \Phi^{\prime}\left(y_{x}\right) \leqslant 2 \Phi\left(y_{x}\right)-y_{x} \Phi^{\prime}\left(y_{x}\right) \leqslant \frac{1-\varepsilon}{1+\varepsilon} y_{x} \Phi^{\prime}\left(y_{x}\right),
$$

then using the last inequality and again the assumption $(\mathbf{H})$ one get

$$
\frac{1}{8 C_{h}(1-\varepsilon)(2-\varepsilon)} \Phi^{\prime 2}\left(y_{x}\right) \leqslant x \leqslant \frac{1}{8 C_{h} \varepsilon(1+\varepsilon)} \Phi^{\prime 2}\left(y_{x}\right) .
$$

We get with the assumption $(\mathbf{H})$,

$$
\begin{aligned}
\forall x \geqslant D, \sup _{y \geqslant m}\{x y-\tau(y)\} & \leqslant \frac{1}{8 C_{h} \varepsilon(1+\varepsilon)} \Phi^{\prime 2}\left(y_{x}\right) \frac{y_{x}^{2}}{\Phi\left(y_{x}\right)}-\frac{1}{8 C_{y}} \Phi\left(y_{x}\right) \\
& \leqslant \frac{(2-\varepsilon)^{2}}{8 C_{h} \varepsilon(1+\varepsilon)} \Phi\left(y_{x}\right) .
\end{aligned}
$$

Equation (2.10) gives,

$$
y_{x} \leqslant \Phi^{\prime-1}(\sqrt{C x})
$$

where $C>0$. Then we get

$$
\forall x \geqslant D, \quad \sup _{y \geqslant m}\{x y-\tau(y)\} \leqslant \frac{(2-\varepsilon)^{2}}{8 C_{h} \varepsilon(1+\varepsilon)} \Phi\left(\Phi^{\prime-1}(\sqrt{C x})\right) .
$$

We obtain, using inequality (2.2) of Lemma 2.1,

$$
\forall x \geqslant D, \quad \sup _{y \geqslant m}\{x y-\tau(y)\} \leqslant \frac{1}{8 C_{h} \varepsilon^{2}(1+\varepsilon)} \Phi^{*}(\sqrt{C x}) .
$$

then,

$$
\forall x \geqslant D, \quad \tau^{*}(x) \leqslant x m+K \Phi^{*}(\sqrt{C x}) .
$$

Using inequality (2.1) of Lemma 2.1 we get

$$
\forall x \geqslant D, \tau^{*}(x) \leqslant K^{\prime} \Phi^{*}(\sqrt{C x}),
$$

for some $K^{\prime} \geqslant 0$.

On the other hand, the function $\tau$ is non-negative and satisfy $\tau(0)=0$ then $\tau^{*}(0)=0 . \tau^{*}$ is also a convex function, then there exists $m^{\prime}$ such that

$$
\forall x \in[0, D], \quad \tau^{*}(x) \leqslant x m^{\prime},
$$

which proves the lemma. 
Corollary 2.6 For any smooth functions $f>0$ on $\mathbb{R}$ satisfying

$$
\int f^{2} d \mu_{\Phi}=1, \text { and } \mathbf{E n t}_{\mu_{\Phi}}\left(f^{2}\right) \geqslant 1
$$

we have

$$
\text { Ent }_{\mu_{\Phi}}\left(f^{2}\right) \leqslant C \int H_{\Phi}\left(\frac{f^{\prime}}{f}\right) f^{2} d \mu_{\Phi}
$$

where

$$
H_{\Phi}(x)=\left\{\begin{aligned}
x^{2} & \text { if }|x|<D \\
\Phi^{*}(B x) & \text { if }|x| \geqslant D
\end{aligned}\right.
$$

and $B, D \geqslant 0$.

Proof. Due to the property $(\mathbf{H})$ the measure $\mu_{\Phi}$ satisfies a Spectral Gap inequality,

$$
\operatorname{Var}_{\mu_{\Phi}}(f) \leqslant C_{S G} \int f^{\prime 2} d \mu_{\Phi}
$$

with $C_{S G} \geqslant 0$. We apply inequality (2.7) to get the result.

\subsection{Small entropy}

Lemma 2.7 Let $\lambda>0$ and define the function $\psi$ by

$$
\psi(x)=\left\{\left(\Phi^{*}\right)^{-1}(\lambda \log x)\right\}^{2} .
$$

Then for all $\lambda>0$ there exists $A_{\lambda}>0$ such that the function $\psi$ is well defined, positive, increasing, concave on $\left[A_{\lambda}, \infty\left[\right.\right.$ and satisfies $\psi\left(A_{\lambda}\right) \geqslant 1$.

Proof. Let $\lambda>0$ be fixed. Classical property of the Legendre-Frenchel transform implies that $\Phi^{*}$ is convex. Due to the property $(\mathbf{H}),\left(\Phi^{*}\right)^{-1}(\lambda \log x)$ is well defined for $x \geqslant M_{1}$ with $M_{1}>0$. Then we get on $\left[M_{1}, \infty\right.$ [,

$$
\begin{aligned}
\psi^{\prime}(x) & =2 g^{\prime}(\lambda \log x) g(\lambda \log x) \frac{\lambda}{x}, \\
\psi^{\prime \prime}(x) & =2 g(\lambda \log x) \frac{\lambda^{2}}{x^{2}}\left(g^{\prime \prime}(\lambda \log x)-\frac{g^{\prime}(\lambda \log x)}{\lambda}+\frac{g^{\prime 2}(\lambda \log x)}{g(\lambda \log x)}\right),
\end{aligned}
$$

where, for simplicity, we have noted $g=\left(\Phi^{*}\right)^{-1}$.

For $x$ large enough $g$ is non-negative and increasing and then $\psi$ is increasing on $\left[M_{2}, \infty\left[\right.\right.$, with $M_{2} \geqslant 0$.

An easy estimation gives that as $x$ goes to infinity,

$$
\frac{g^{\prime}(x)}{g(x)}=o_{\infty}(1)
$$

then since $\left(\Phi^{*}\right)^{-1}$ is concave, for all large enough $x, \psi^{\prime \prime}(x) \leqslant 0$. Then one can find $A_{\lambda}>0$ such that properties on the Lemma 2.7 are true. 
The proof of $M L S I$ for small entropy is based on the next lemma, we give a $M L S I$ saturate on the right.

Lemma 2.8 Let $T_{1}, T_{2} \in \mathbb{R}$ such that $T_{1}<T_{2}$. Then there exists $\lambda>0$ which depends on the function $\Phi$ such that for all $g$ defined on $[T, \infty[$ with $T \in\left[T_{1}, T_{2}\right]$, and verifying that

$$
g(T)=\sqrt{A_{\lambda}}, g \geqslant \sqrt{A_{\lambda}} \text { and } \int_{T}^{\infty} g^{2} d \mu_{\alpha} \leqslant 2 A_{\lambda}+2,
$$

where $A_{\lambda}$ is defined on Lemma 2.7.

Then we get

$$
\int_{T}^{\infty}\left(g-\sqrt{A_{\lambda}}\right)_{+}^{2} \psi\left(g^{2}\right) \mu_{\Phi} \leqslant C_{1} \int_{[T, \infty[} g^{\prime 2} d \mu_{\Phi},
$$

where $\psi$ is defined on Lemma 2.7.

The constant $C_{1}$ depends on $\Phi$ and $\lambda$ but does not depend on the value of $T \in\left[T_{1}, T_{2}\right]$.

Proof. Let use Hardy's inequality as explained in the introduction. We have $g(T)=A_{\lambda}$. We apply inequality (1.12) on $[T, \infty[$ with the function $\left(g-\sqrt{A_{\lambda}}\right)+$ and the following measures

$$
d \mu=\psi\left(g^{2}\right) d \mu_{\Phi} \text { and } \nu=\mu_{\Phi} .
$$

Then the constant $C$ in inequality (2.12) is finite if and only if

$$
B=\sup _{x \geqslant T} \int_{T}^{x} e^{\Phi(t)} d t \int_{x}^{\infty} \psi\left(g^{2}\right) d \mu_{\Phi}
$$

is finite.

By Lemma $2.7, \psi$ is concave on $\left[A_{\lambda}, \infty[\right.$ then by Jensen inequality, for all $x \geqslant T$ we get

$$
\int_{x}^{\infty} \psi\left(g^{2}\right) d \mu_{\Phi} \leqslant \mu_{\Phi}\left(\left[x, \infty[) \psi\left(\frac{\int_{x}^{\infty} g^{2} d \mu_{\Phi}}{\mu_{\Phi}([x, \infty[)}\right) .\right.\right.
$$

Then we have

$$
B \leqslant \sup _{x>T_{1}}\left\{\int _ { T _ { 1 } } ^ { x } e ^ { \Phi ( t ) } d t \mu _ { \Phi } \left(\left[x, \infty[) \psi\left(\frac{\int_{x}^{\infty} g^{2} d \mu_{\Phi}}{\mu_{\Phi}([x, \infty[)}\right)\right\}\right.\right.
$$

Due to the property $(\mathbf{H})$ there exists $K>1$ such that

$$
\Phi^{\prime}(x) e^{\Phi(x)} \leqslant e^{K \Phi(x)},
$$

and

for large enough $x$.

$$
\int_{T_{1}}^{x} e^{\Phi(t)} d t \leqslant \frac{K e^{\Phi(x)}}{\Phi^{\prime}(x)}, \quad \int_{x}^{\infty} e^{-\Phi(t)} d t \leqslant \frac{e^{-\Phi(x)}}{\Phi^{\prime}(x)},
$$


By (2.14) we get also for large enough $x$ that

$$
\frac{e^{-K \Phi(x)}}{Z_{\Phi}} \leqslant \frac{\int_{x}^{\infty} e^{-\Phi(t)} d t}{Z_{\Phi}}
$$

Then for large enough $x$, uniformly in the previous $g$, one have

$$
\int_{T_{1}}^{x} e^{\Phi(t)} d t \mu_{\Phi}\left(\left[x, \infty[) \psi\left(\frac{\int_{x}^{\infty} g^{2} d \mu_{\Phi}}{\mu_{\Phi}([x, \infty[)}\right) \leqslant \frac{K}{\left(\Phi^{\prime}(x)\right)^{2}} \psi\left(\frac{\int_{x}^{\infty} g^{2} d \mu_{\Phi}}{K} e^{K \Phi(x)}\right) .\right.\right.
$$

For $x$ large enough,

$$
\frac{\int_{x}^{\infty} g^{2} d \mu_{\Phi}}{K} \leqslant 1
$$

Then, by definition of $\psi$, for large enough $x$,

$$
\int_{T_{1}}^{x} e^{\Phi(t)} d t \mu_{\Phi}\left(\left[x, \infty[) \psi\left(\frac{\int_{x}^{\infty} g^{2} d \mu_{\Phi}}{\mu_{\Phi}([x, \infty[)}\right) \leqslant K\left(\frac{\Phi^{*-1}(\lambda K \Phi(x))}{\Phi^{\prime}(x)}\right)^{2} .\right.\right.
$$

There is also $C_{\epsilon}$ such that, for $x$ large enough

$$
\Phi^{*-1}(x) \leqslant \Phi^{\prime}\left(\Phi^{-1}\left(C_{\epsilon} x\right)\right),
$$

as one can see from equation $(2.2)$.

Then one can choose $\lambda=1 /\left(K C_{\epsilon}\right)$ and the lemma is proved. Note that $\lambda$ depends only on the function $\Phi$.

The constant $B$ on (2.13) is bounded by $K$ which does not depend on $T$ on $\left[T_{1}, T_{2}\right]$.

Proposition 2.9 There exists $A, A^{\prime}, B, D, A_{\lambda}>0$ such that for any functions $f \geqslant 0$ satisfying

$$
\int f^{2} d \mu_{\alpha}=1 \text { and } \operatorname{Ent}_{\mu_{\alpha}}\left(f^{2}\right) \leqslant 1
$$

we have

$$
\text { Ent }_{\mu_{\Phi}}\left(f^{2}\right) \leqslant A \operatorname{Var}_{\mu_{\Phi}}(f)+A^{\prime} \int_{f^{2} \geqslant A_{\lambda}} H_{\Phi}\left(\frac{f^{\prime}}{f}\right) f^{2} d \mu_{\Phi}
$$

where

$$
H_{\Phi}(x)=\left\{\begin{aligned}
x^{2} & \text { if }|x|<D \\
\Phi^{*}(B x) & \text { if }|x| \geqslant D .
\end{aligned}\right.
$$

Note that contrary to Proposition 2.4, constant $A$ depends on the function $\Phi$. 
Proof. Let $f \geqslant 0$ satisfying $\int f^{2} d \mu_{\alpha}=1$.

We can assume that $A_{\lambda} \geqslant 2$. A careful study of the function

$$
x \rightarrow-x^{2} \log x^{2}+A(x-1)^{2}+x^{2}-1+\left(x-\sqrt{A_{\lambda}}\right)_{+}^{2} \log \left(x-\sqrt{A_{\lambda}}\right)_{+}^{2}
$$

proves that there exists $A$ such that for every $x \in \mathbb{R}^{+}$

$$
x^{2} \log x^{2} \leqslant A(x-1)^{2}+x^{2}-1+\left(x-\sqrt{A_{\lambda}}\right)_{+}^{2} \log \left(x-\sqrt{A_{\lambda}}\right)_{+}^{2} .
$$

Then we get

$$
\text { Ent }_{\mu_{\alpha}}\left(f^{2}\right)=\int f^{2} \log f^{2} d \mu_{\alpha} \leqslant A \operatorname{Var}_{\mu_{\alpha}}(f)+\int\left(f-\sqrt{A_{\lambda}}\right)_{+}^{2} \log f^{2} d \mu_{\alpha}
$$

where $\sqrt{A_{\lambda}}$ is defined as in Lemma 2.8 .

Fix $\lambda$ as in Lemma 2.8. We define the function $K$ on $\left[A_{\lambda}, \infty[\right.$ by

$$
K(x)=\sqrt{\frac{\log x^{2}}{\psi\left(x^{2}\right)}} .
$$

Let now define $T_{1}<T_{2}$ such that

$$
\left.\left.\mu_{\Phi}(] \infty, T_{1}\right]\right)=\frac{3}{8}, \mu_{\Phi}\left(\left[T_{1}, T_{2}\right]\right)=\frac{1}{4} \text { and } \mu_{\Phi}\left(\left[T_{2},+\infty[)=\frac{3}{8} .\right.\right.
$$

Since $\int f^{2} d \mu_{\Phi}=1$ there exists $T \in\left[T_{1}, T_{2}\right]$ such that $f(T) \leqslant A_{\lambda}$.

Let us define $g$ on $[T, \infty]$ as follow

$$
g=\sqrt{A_{\lambda}}+\left(f-\sqrt{A_{\lambda}}\right)_{+} K(f) \text { on }[T, \infty[.
$$

Function $g$ satisfies $g(T)=\sqrt{A_{\lambda}}$ and $g(x) \geqslant \sqrt{A_{\lambda}}$ for all $x \geqslant T$. Then we have

$$
\begin{aligned}
\int_{T}^{\infty} g^{2} d \mu_{\Phi} & \leqslant 2 A_{\lambda}+2 \int_{\left[T_{1}, \infty\left[\cap\left\{f^{2} \geqslant A_{\lambda}\right\}\right.\right.} f^{2} K^{2}(f) d \mu_{\Phi} \\
& \leqslant 2 A_{\lambda}+2 \int_{\left[T_{1}, \infty[\right.} f^{2} \log \left(f^{2}\right) d \mu_{\Phi} \\
& \leqslant 2 A_{\lambda}+2,
\end{aligned}
$$

where we are using the growth of $\psi$ on $\left[A_{\lambda}, \infty\left[\right.\right.$ and $\psi\left(A_{\lambda}\right) \geqslant 1$.

Assumptions on Lemma 2.8 are satisfied, we obtain by inequality $(2.12)$

$$
\int_{T}^{\infty}\left(g-\sqrt{A_{\lambda}}\right)_{+}^{2} \psi\left(g^{2}\right) d \mu_{\Phi} \leqslant C_{1} \int_{\left[T, \infty\left[\cap\left\{f^{2} \geqslant A_{\lambda}\right\}\right.\right.} g^{\prime 2} d \mu_{\Phi}
$$

Let us compare the various terms now. 
Due to the property $(\mathbf{H}), K$ is lower bounded on $\left[\sqrt{A_{\lambda}}, \infty[\right.$ by $\alpha \geqslant 1$ (maybe for $A_{\lambda}$ larger), then we get firstly

$\sqrt{A_{\lambda}}+\left(f-\sqrt{A_{\lambda}}\right)_{+} K(f) \geqslant \sqrt{A_{\lambda}}+\left(f-\sqrt{A_{\lambda}}\right)_{+} \alpha \geqslant f$ on $\left\{f^{2} \geqslant A_{\lambda}\right\}$.

Then

$$
\left(g-\sqrt{A_{\lambda}}\right)_{+}^{2} \psi\left(g^{2}\right) \geqslant\left(f-\sqrt{A_{\lambda}}\right)_{+}^{2} K(f)^{2} \psi\left(f^{2}\right)=\left(f-\sqrt{A_{\lambda}}\right)_{+}^{2} \log f^{2},
$$

by the definition of $K$, then we obtain

$$
\int_{T}^{\infty}\left(f-\sqrt{A_{\lambda}}\right)_{+}^{2} \log f^{2} d \mu_{\Phi} \leqslant \int_{T}^{\infty}\left(g-\sqrt{A_{\lambda}}\right)_{+}^{2} \psi\left(g^{2}\right) d \mu_{\Phi} .
$$

Secondly we have on $\left\{f \geqslant \sqrt{A_{\lambda}}\right\}$

$$
\begin{aligned}
g^{\prime} & =f^{\prime} K(f)+\left(f-\sqrt{A_{\lambda}}\right)_{+} f^{\prime} K^{\prime}(f) \\
& =f^{\prime} K(f)\left(1+\left(f-\sqrt{A_{\lambda}}\right)_{+} \frac{K^{\prime}(f)}{K(f)}\right) .
\end{aligned}
$$

But we have, for $x \geqslant \sqrt{A_{\lambda}}$,

$$
\begin{aligned}
\left|1+\left(x-\sqrt{A_{\lambda}}\right) \frac{K^{\prime}(x)}{K(x)}\right| & \leqslant 1+x\left|\frac{K^{\prime}(x)}{K(x)}\right| \\
& \leqslant 1+\frac{1}{\log x}+4\left|\frac{\lambda}{x} \frac{g^{\prime}(\lambda 2 \log x)}{g(\lambda 2 \log x)}\right|
\end{aligned}
$$

where $g(x)=\Phi^{*-1}(x)$. Using the estimation (2.11) we obtain that there exists $C>0$ such that for all $x \geqslant \sqrt{A_{\lambda}}$,

$$
\left|1+\left(x-\sqrt{A_{\lambda}}\right) \frac{K^{\prime}(x)}{K(x)}\right| \leqslant C .
$$

We get then

$$
g^{\prime 2} \leqslant C f^{\prime 2} K^{2}(f) \quad \text { on } \quad\left\{f^{2} \geqslant A_{\lambda}\right\}
$$

for some $C<\infty$ and then

$$
\int_{\left[T, \infty\left[\cap\left\{f^{2} \geqslant A_{\lambda}\right\}\right.\right.} g^{\prime 2} d \mu_{\Phi} \leqslant C \int_{\left[T, \infty\left[\cap\left\{f^{2} \geqslant A_{\lambda}\right\}\right.\right.} f^{\prime 2} K^{2}(f) d \mu_{\Phi} .
$$

By equation (2.17) and (2.18) we obtain

$$
\int_{T}^{\infty}\left(f-\sqrt{A_{\lambda}}\right)_{+}^{2} \log f^{2} d \mu_{\Phi} \leqslant C \int_{\left[T, \infty\left[\cap\left\{f^{2} \geqslant A_{\lambda}\right\}\right.\right.} f^{\prime 2} K^{2}(f) d \mu_{\Phi} .
$$


Let $u_{0}>0$,

$$
\begin{gathered}
\int_{T}^{\infty}\left(f-\sqrt{A_{\lambda}}\right)_{+}^{2} \log f^{2} d \mu_{\Phi} \leqslant \\
C \int_{\left[T, \infty\left[\cap\left\{f^{2} \geqslant A_{\lambda}\right\}\right.\right.} \tau_{2}^{*}\left(u_{0}\left(\frac{f^{\prime}}{f}\right)^{2}\right) f^{2} d \mu_{\Phi}+\int_{\left[T, \infty\left[\cap\left\{f^{2} \geqslant A_{\lambda}\right\}\right.\right.} \tau_{2}\left(\frac{K^{2}(f)}{u_{0}}\right) f^{2} d \mu_{\Phi},
\end{gathered}
$$

where the function $\tau_{2}$ is defined as in equation (2.9) by

$$
\tau_{2}(x)=\left\{\begin{array}{lr}
x \Phi\left(h^{-1}(m)\right) \frac{1-\epsilon}{2 \lambda m} & \text { if } 0 \leqslant x<m \\
\Phi\left(h^{-1}(x)\right) \frac{1-\epsilon}{2 \lambda} & \text { if } x \geqslant m
\end{array}\right.
$$

where $h$ is defined on equation (2.5) and $m$ on equation (2.9). The function $\tau_{2}$ is equal to $\tau$ up to a constant factor.

Using Lemma 2.10 we get

$$
\begin{aligned}
& \int_{T}^{\infty}\left(f-\sqrt{A_{\lambda}}\right)_{+}^{2} \log f^{2} d \mu_{\Phi} \leqslant \\
& \quad C \int_{\left[T, \infty\left[\cap\left\{f^{2} \geqslant A_{\lambda}\right\}\right.\right.} \tau_{2}^{*}\left(u_{0}\left(\frac{f^{\prime}}{f}\right)^{2}\right) f^{2} d \mu_{\Phi}+\frac{1}{2} \int_{\left[T, \infty\left[\cap\left\{f^{2} \geqslant A_{\lambda}\right\}\right.\right.} f^{2} \log f^{2} d \mu_{\Phi} .
\end{aligned}
$$

The same method can be used on $]-\infty, T]$ and then there is $C^{\prime}<\infty$ such that

$$
\begin{gathered}
\int_{-\infty}^{T}\left(f-\sqrt{A_{\lambda}}\right)_{+}^{2} \log f^{2} d \mu_{\Phi} \leqslant \\
C^{\prime} \int_{]-\infty, T] \cap\left\{f^{2} \geqslant A_{\lambda}\right\}} \tau_{2}^{*}\left(u_{0}\left(\frac{f^{\prime}}{f}\right)^{2}\right) f^{2} d \mu_{\Phi}+\frac{1}{2} \int_{]-\infty, T] \cap\left\{f^{2} \geqslant A_{\lambda}\right\}} f^{2} \log f^{2} d \mu_{\Phi}
\end{gathered}
$$

And then we get

$$
\begin{aligned}
& \int\left(f-\sqrt{A_{\lambda}}\right)_{+}^{2} \log f^{2} d \mu_{\Phi} \leqslant \\
&\left(C+C^{\prime}\right) \int_{\left\{f^{2} \geqslant A_{\lambda}\right\}} \tau_{2}^{*}\left(u_{0}\left(\frac{f^{\prime}}{f}\right)^{2}\right) f^{2} d \mu_{\Phi}+\frac{1}{2} \int_{\left\{f^{2} \geqslant A_{\lambda}\right\}} f^{2} \log f^{2} d \mu_{\Phi} .
\end{aligned}
$$

Note that constants $C$ and $C^{\prime}$ do not depend on $T \in\left[T_{1}, T_{2}\right]$.

Then by inequality (2.15) and Lemma 2.5, Proposition 2.9 is proved. 
Lemma 2.10 There exists $u_{0}>0$ such that, for all $x \geqslant A_{\lambda}$ we have

$$
\tau_{2}\left(\frac{K^{2}(x)}{u_{0}}\right) \leqslant \frac{1}{2} \log x^{2}
$$

Proof. Let $\kappa=2 \lambda /(1-\epsilon)$. For all $x \geqslant M$, where $M$ is defined on equation (2.9), we have

$$
\begin{gathered}
\tau_{2}(h(x))=\frac{\Phi(x)}{\kappa}, \\
\tau_{2}\left(\frac{x^{2}}{\Phi(x)}\right)=\frac{\Phi(x)}{\kappa} .
\end{gathered}
$$

$\tau_{2}$ is increasing, then due to the property $(\mathbf{H})$ we have for $x \geqslant M$

$$
\tau_{2}\left((1+\epsilon)^{2} \frac{\Phi(x)}{\Phi^{\prime}(x)^{2}}\right) \leqslant \frac{\Phi(x)}{\kappa} .
$$

Using now inequality (2.2) one has

$$
\frac{1}{\Phi^{\prime}(x)} \geqslant \frac{1}{\Phi^{*-1}((1-\epsilon) \Phi(x))}
$$

then for all $x \geqslant M$,

$$
\tau_{2}\left((1+\epsilon)^{2} \frac{\Phi(x)}{\Phi^{*-1}((1-\epsilon) \Phi(x))^{2}}\right) \leqslant \frac{\Phi(x)}{\kappa} .
$$

Take now $z=(1-\epsilon) \Phi(x)$,

$$
\tau_{2}\left(\frac{(1+\epsilon)^{2}}{1-\epsilon} \frac{z}{\Phi^{*-1}(z)^{2}}\right) \leqslant \frac{z}{(1-\epsilon) \kappa},
$$

to finish take

$$
x=\exp \left(\frac{z}{(1-\epsilon) \kappa}\right)
$$

to obtain

$$
\tau_{2}\left((1+\epsilon)^{2} \kappa \frac{\log x^{2}}{\Phi^{*-1}\left(\frac{(1-\epsilon) \kappa}{2} \log x^{2}\right)^{2}}\right) \leqslant \frac{1}{2} \log x^{2} .
$$

Recall that $\lambda=(1-\epsilon) \kappa / 2$ and let take $u_{0}=1 /\left((1+\epsilon)^{2} \kappa\right)$, to obtain the result for $x \geqslant C$, where $C$ is a constant depending on $\Phi$.

If we have $A_{\lambda}<C$, one can change the value of $u_{0}$ to obtain also the result on $\left[A_{\lambda}, C\right]$. 
Proof of Theorem 1.2. To give the proof of the theorem we need to give an other result like Proposition 2.4. By the same argument as in Proposition 2.4 one can also prove that there exists $A, A^{\prime}, B, D>0$ such that for any functions $f \geqslant 0$ satisfying

$$
\int f^{2} d \mu_{\alpha}=1 \text { and } \operatorname{Ent}_{\mu_{\alpha}}\left(f^{2}\right) \geqslant 1
$$

we have for some $C^{\prime}\left(A_{\lambda}\right), C\left(A_{\lambda}\right)$

$$
\operatorname{Ent}_{\mu_{\Phi}}\left(f^{2}\right) \leqslant C^{\prime}\left(A_{\lambda}\right) \operatorname{Var}_{\mu_{\Phi}}(f)+C\left(A_{\lambda}\right) \int_{f^{2} \geqslant A_{\lambda}} H_{\Phi}\left(\frac{f^{\prime}}{f}\right) f^{2} d \mu_{\Phi}
$$

where $H_{\Phi}$ is defined on (1.10) and $A_{\lambda}$ on the Proposition 2.9.

Then the proof of the theorem is a simple consequence of (2.20) and Proposition 2.9.

\section{Classical properties and applications}

Let us give here properties inherited directly from the methodology known for classical logarithmic Sobolev inequalities.

Proposition 3.1 1 This property is known under the name of tensorization.

Let $\mu_{1}$ and $\mu_{2}$ two probability measures on $\mathbb{R}^{n_{1}}$ and $\mathbb{R}^{n_{2}}$. Suppose that $\mu_{1}$ (resp. $\mu_{2}$ ) satisfies the a MLSI with function $H_{\Phi}$ and constant $A_{1}$ (resp. with constant $A_{2}$ ) then the probability $\mu_{1} \otimes \mu_{2}$ on $\mathbb{R}^{n_{1}+n_{2}}$, satisfies a MLSI with function $H_{\Phi}$ and constant $\max \left\{A_{1}, A_{2}\right\}$.

2 This property is known under the name of perturbation.

Let $\mu$ a measure on $\mathbb{R}^{n}$ a MLSI with function $H_{\Phi}$ and constant $A$. Let $h$ a bounded function on $\mathbb{R}^{n}$ and defined $\tilde{\mu}$ as

$$
d \tilde{\mu}=\frac{e^{h}}{Z} d \mu
$$

where $Z=\int e^{h} d \mu$.

Then the measure $\tilde{\mu}$ satisfies a MLSI with function $H_{\Phi}$ and the constant

$$
D=A e^{2 o s c(h)}, \quad \text { where osc }(h)=\sup (h)-\inf (h) .
$$


3 Link between MLSI of function $H_{\Phi}$ with Poincaré inequality.

Let $\mu$ a measure on $\mathbb{R}^{n}$. If $\mu$ satisfies a MLSI with function $H_{\Phi}$ and constant $A$, then $\mu$ satisfies a Poincaré inequality with the constant $A$. Let us recall that $\mu$ satisfies a Poincaré inequality with constant $A$ if

$$
\operatorname{Var}_{\mu}(f) \leqslant A \int|\nabla f|^{2} d \mu
$$

for all smooth function $f$.

Proof. One can find the details of the proof of the properties of tensorization and perturbation and the implication of the Poincaré inequality in chapters 1 and 3 of [1, Section 1.2.6., Theorem 3.2.1 and Theorem 3.4.3].

Proposition 3.2 Assume that the probability measure $\mu$ on $\mathbb{R}$ satisfies a $M L S I$ with function $H_{\Phi}$ and constant $A$. Then there exists three constants $B, C, D \geqslant 0$, independent of $n$ such that: if $F$ is a function on $\mathbb{R}^{n}$ such that $\forall i,\left\|\partial_{i} F\right\|_{\infty} \leqslant \zeta$, then we get for $\lambda \geqslant 0$,

$$
\begin{aligned}
\mu^{\otimes n}\left(\left|F-\mu^{\otimes n}(F)\right| \geqslant \lambda\right) & \begin{cases}2 \exp \left(-n B \Phi\left(C \frac{\lambda}{n \zeta}\right)\right) & \text { if } \lambda>n D \zeta \\
2 \exp \left(-B \frac{\lambda^{2}}{n \zeta^{2}}\right) & \text { if } 0 \leqslant \lambda \leqslant n D \zeta\end{cases}
\end{aligned}
$$

Proof. Let us first present the proof when $n=1$. Assume, without loss of generality, that $\int F d \mu=0$. Due to the homogeneous property of (3.1) on can suppose that $\zeta=1$.

Let us recall briefly Herbst's argument (see [1, Chapter 7] for more details). Denote $\psi(t)=\int e^{t F} d \mu$, and remark that $M L S I$ of function $H_{\Phi}$ applied to $f^{2}=e^{t F}$, using basic properties of $H_{\Phi}$, yields to

$$
t \psi^{\prime}(t)-\psi(t) \log \psi(t) \leq A H_{\Phi}\left(\frac{t}{2}\right) \psi(t)
$$

which, denoting $K(t)=(1 / t) \log \psi(t)$, entails

$$
K^{\prime}(t) \leqslant \frac{A}{t^{2}} H_{\Phi}\left(\frac{t}{2}\right)
$$

Then, integrating, and using $K(0)=\int F d \mu=0$, we obtain

$$
\psi(t) \leq \exp \left(A t \int_{0}^{t} \frac{1}{s^{2}} H_{\Phi}\left(\frac{s}{2}\right) d s\right) .
$$


Then we get using Markov inequality

$$
\mu(|F-\mu(F)| \geqslant \lambda) \leqslant 2 \exp \left(\min _{t \geqslant 0}\left\{A t \int_{0}^{t} \frac{1}{s^{2}} H_{\Phi}\left(\frac{s}{2}\right) d s-\lambda t\right\}\right) .
$$

Let note, for $t \geqslant 0$,

$$
G(t)=A t \int_{0}^{t} \frac{1}{s^{2}} H_{\Phi}\left(\frac{s}{2}\right) d s-\lambda t .
$$

An easy study proves that $G$ admits a minimum on $\mathbb{R}^{+}$on the value $t_{0}$ which satisfies for $\lambda>0 G^{\prime}\left(t_{0}\right)=0$. Then due to the definition of $H_{\Phi}$ we get that

$$
\min _{t \geqslant 0}\{G(t)\}=-\frac{\lambda^{2}}{A}, \quad \text { if } \lambda \leqslant A D .
$$

Assume now that $\lambda \geqslant A D$ then we obtain after derivation

$$
\min _{t \geqslant 0}\{G(t)\}=-A \Phi^{*}\left(t_{0} \frac{B}{2}\right)
$$

with

$$
\lambda t_{0}=A t_{0} \int_{0}^{t_{0}} \frac{1}{s^{2}} H_{\Phi}\left(\frac{s}{2}\right) d s+A H_{\Phi}\left(\frac{t_{0}}{2}\right) .
$$

We first prove that there exists $C \geqslant 0$ such that for all $t_{0}$ large enough

$$
t_{0} \int_{0}^{t_{0}} \frac{1}{s^{2}} H_{\Phi}\left(\frac{s}{2}\right) d s \leqslant C H_{\Phi}\left(\frac{t_{0}}{2}\right) .
$$

For $\kappa \geqslant 0$ large enough and $t_{0} \geqslant \kappa$ we get using then inequality (2.2) we get

$$
t_{0} \int_{\kappa}^{t_{0}} \frac{1}{s^{2}} H_{\Phi}\left(\frac{s}{2}\right) d s \leqslant C t_{0} \int_{\kappa}^{t_{0}} \frac{1}{s^{2}} \Phi\left(\Phi^{\prime-1}\left(\frac{s}{2}\right)\right) d s,
$$

with $C \geqslant 0$. Then by a change of variables and integration by parts, for large enough $t_{0}$,

$$
\begin{aligned}
t_{0} \int_{\kappa}^{t_{0}} \frac{1}{s^{2}} \Phi\left(\Phi^{\prime-1}\left(\frac{s}{2}\right)\right) d s & =\frac{t_{0}}{2} \int_{\Phi^{\prime-1}\left(\frac{\kappa}{2}\right)}^{\Phi^{\prime-1}\left(\frac{t_{0}}{2}\right)} \frac{\Phi(u)}{\Phi^{\prime}(u)^{2}} \Phi^{\prime \prime}(u) d u \\
& \leqslant \frac{t_{0}}{2} \frac{\Phi\left(\Phi^{-1}(\kappa / 2)\right)}{\Phi^{\prime}\left(\Phi^{-1}(\kappa / 2)\right)}+\frac{t_{0}}{2} \Phi^{\prime-1}\left(t_{0} / 2\right) \\
& \leqslant C t_{0} \Phi^{\prime-1}\left(t_{0} / 2\right),
\end{aligned}
$$

for some other $C \geqslant 0$. Then we get, using inequality (2.3), for $t_{0}$ large enough,

$$
t_{0} \int_{0}^{t_{0}} \frac{1}{s^{2}} H_{\Phi}\left(\frac{s}{2}\right) d s \leqslant C t_{0} \Phi^{\prime-1}\left(t_{0} / 2\right) \leqslant C^{\prime} \Phi^{*}\left(t_{0} / 2\right) .
$$


for some constant $C^{\prime} \geqslant 0$ and for $t_{0}$ large enough. Then inequality (3.5) is proved. By (3.5) and (3.4) one get for $t_{0}$ large enough,

$$
\lambda t_{0} \leqslant A^{\prime} \Phi^{*}\left(\frac{t_{0}}{2}\right)
$$

for some constant $A^{\prime} \geqslant 0$. But, using inequality (2.3) and property (H) we get then, for other constants $C, C^{\prime}, A$,

$$
\begin{gathered}
\Phi^{\prime}(A \lambda) \leqslant C t_{0} \\
\min _{t \geqslant 0}\{G(t)\} \leqslant-A \Phi^{*}\left(B \Phi^{\prime}(C \lambda)\right) \leqslant-A \Phi^{*}\left(\Phi^{\prime}\left(C^{\prime} \lambda\right)\right),
\end{gathered}
$$

if $\lambda$ is large enough and for some other constants $A, B, C, C^{\prime} \geqslant 0$. Using inequality (2.2), we obtain the result in dimension 1 .

For the $n$-dimensional extension, use the tensorization property of $M L S I$ of function $H_{\Phi}$ and

$$
\sum_{i=1}^{n} H_{\Phi}\left(\frac{t}{2} \partial_{i} F\right) \leqslant n H_{\Phi}\left(\frac{t}{2}\right)
$$

Then we can use the case of dimension 1 with the constant $A$ replaced by $A n$.

Remark 3.3 Let us present a simple application of the preceding proposition to deviation inequality of the empirical mean of a function. Consider the real valued function $f$, with $\left|f^{\prime}\right| \leq 1$. Let apply Proposition 3.2 with the two functions

$$
F\left(x_{1}, \ldots, x_{n}\right)=\frac{1}{n} \sum_{k=1}^{n} f\left(x_{k}\right) \quad \text { and } \quad F\left(x_{1}, \ldots, x_{n}\right)=\frac{1}{\sqrt{n}} \sum_{k=1}^{n} f\left(x_{i}\right) .
$$

We obtain then

$$
\begin{gathered}
\mathbb{P}\left(\frac{1}{n}\left|\sum_{k=1}^{n} f\left(X_{k}\right)-\mu(f)\right|>\lambda\right) \leqslant \begin{cases}2 \exp (-n A \Phi(B \lambda)) & \text { if } \lambda \geqslant D, \\
2 \exp \left(-n A \lambda^{2}\right) & \text { if } 0 \leqslant \lambda \leqslant D,\end{cases} \\
\mathbb{P}\left(\frac{1}{\sqrt{n}}\left|\sum_{k=1}^{n} f\left(X_{k}\right)-\mu(f)\right|>\lambda\right) \\
\leqslant \begin{cases}2 \exp \left(-n A \Phi\left(B \frac{\lambda}{\sqrt{n}}\right)\right) & \text { if } \lambda \geqslant D \sqrt{n}, \\
2 \exp \left(-A \lambda^{2}\right) & \text { if } 0 \leqslant \lambda \leqslant D \sqrt{n} .\end{cases}
\end{gathered}
$$




\section{References}

[1] Ané, C., Blachère, S., Chafaï, D., Fougères, P., Gentil, I., Malrieu, F., Roberto, C. And Scheffer, G.: Sur les inégalités de Sobolev logarithmiques. Panoramas et Synthèses 10. Société Mathématique de France, Paris, 2000.

[2] BAKRY, D.: L'hypercontractivité et son utilisation en théorie des semigroupes. In Lectures on probability theory. École d'été de probabilités de St-Flour 1992, 1-114. Lecture Notes in Mathematics 1581. Springer, Berlin, 1994.

[3] BAKry, D. ANd Émery, M.: Diffusions hypercontractives. In Séminaire de probabilités, XIX, 1983/84, 177-206. Lecture Notes in Mathematics 1123. Springer, Berlin, 1985.

[4] Barthe, F., Cattiaux, P. and Roberto, C.: Interpolated inequalities between exponential and Gaussian, Orlicz hypercontractivity and isoperimetry. Rev. Mat. Iberoamericana 22 (2006), no. 3, 993-1067.

[5] Barthe, F. And Roberto, C.: Sobolev inequalities for probability measures on the real line. Studia Math. 159 (2003), 481-497.

[6] Bobkov, S. G. And Götze, F.: Exponential integrability and transportation cost related to logarithmic Sobolev inequalities. J. Funct. Anal. 163 (1999), 1-28.

[7] Bobkov, S. G. And Ledoux, M.: Poincaré's inequalities and Talagrand's concentration phenomenon for the exponential distribution. Probab. Theory Related Fields 107 (1997), 383-400.

[8] Gentil, I., Guillin, A. And Miclo, L.: Modified logarithmic Sobolev inequalities and transportation inequalities. Probab. Theory Related Fields 133 (2005), no. 3, 409-436.

[9] Gross, L.: Logarithmic Sobolev inequalities. Amer. J. Math. 97 (1975), 1061-1083.

[10] Ledoux, M.: Concentration of measure and logarithmic Sobolev inequalities. In Séminaire de Probabilités XXXIII, 120-216. Lecture Notes in Mathematics 1709. Springer, Berlin, 1999.

[11] Ledoux, M.: The concentration of measure phenomenon. Mathematical Surveys and Monographs 89. American Mathematical Society, Providence, RI, 2001.

[12] Muckenhoupt, B.: Hardy's inequality with weights. Collection of articles honoring the completion by Antoni Zygmund of 50 years of scientific activity, I. Studia Math. 44 (1972), 31-38.

[13] Talagrand, M.: Concentration of measure and isoperimetric inequalities in product spaces. Inst. Hautes Études Sci. Publ. Math. 81 (1995), 73-205. 
Recibido: 22 de abril de 2005

Revisado: 8 de noviembre de 2005

\section{Ivan Gentil \\ CEREMADE}

(UMR 7534, Université Paris-Dauphine et CNRS)

Place du Maréchal De Lattre de Tassigny

75775 Paris Cédex 16, France

gentil@ceremade.dauphine.fr

http://www . ceremade.dauphine.fr/ gentil

Arnaud Guillin

Ecole Centrale Marseille et LATP

(UMR 6632, Université de Provence et CNRS)

39, rue F. Joliot Curie

13453 Marseille Cédex 13, France guillin@cmi.univ-mrs.fr

http://www . latp.univ-mrs.fr/ guillin/

Laurent Miclo

Laboratoire d'Analyse, Topologie, et Probabilités

(UMR 6632, Université de Provence et CNRS)

39, rue F. Joliot Curie, 13453 Marseille Cédex 13, France miclo@cmi.univ-mrs.fr 